\title{
The category effect in visual detection and partial report
}

\author{
PETER DIXON \\ University of Alberta, Edmonton, Alberta, Canada
}

\begin{abstract}
The category effect refers to the more efficient processing of a multi-item array that can occur when some of the items are known to be from an irrelevant category, such as letters instead of digits. Previous research has found category effects in visual search, visual detection, and partialreport tasks. The present experiments investigated the category effect in a task that combined elements of both visual detection and partial report. Experiment 1 found a strong category effect when there was only one item in the relevant category; Experiment 2 found no effect at all when there were three items in the relevant category. The results are discussed in terms of two common accounts of the category effect: partial analysis and parallel categorization.
\end{abstract}

A multi-item visual display can often be processed more efficiently when some of the items are known to belong to an irrelevant category, such as letters instead of digits. This "category effect" has been found in visual search (Egeth, Jonides, \& Wall, 1972), in visual detection (Duncan, 1980; Schneider \& Shiffrin, 1977), and in partial report (Duncan, 1983; Merikle, 1980; von Wright, 1972). At least two kinds of explanations of the category effect have been proposed: the partial analysis model and the parallel-categorization model. The present work investigates these explanations with a new paradigm developed by Di Lollo and Moscovitch (1983) which combines aspects of visual detection and partial report. The results pose some additional constraints on how these accounts might operate.

In visual search tasks, subjects are timed while they search for a target in an array of items. Usually, response time increases linearly with the number of nontarget, or distractor, items (e.g., Nickerson, 1966). A common interpretation is that subjects serially consider each item in the array to decide whether it is a target item, and that each additional distractor item in the array adds to the total response time (cf. Atkinson, Holmgren, \& Juola, 1969). However, Egeth et al. (1972) reported that, after practice, the time to find a digit in an array of letters was unaffected by the number of letters. Although a small effect of the number of distractors has been found in other studies, there is little doubt that the search for a digit is faster among letters than it is among other digits (e.g., Brand, 1971; Egeth, Atkinson, Gilmore, \& Marcus, 1973; Gleitman \& Jonides, 1978; Taylor, 1978). Moreover, the effect has been found even when target and distractors

This research was supported by the Natural Sciences and Engineering Research Council of Canada Grant A8263. I would like to thank Vincent Di Lollo for many cogent discussions during the course of this project and for insightful comments on this manuscript. Requests for reprints should be sent to Peter Dixon, Department of Psychology, University of Alberta, Edmonton, Alta., Canada T6G 2E9. are matched for similarity in visual features (Ingling, 1972; Jonides \& Gleitman, 1972; but see Krueger, 1984).

A similar finding occurs in visual detection tasks. In this case, subjects are asked to detect the presence of a target item in a briefly presented array of items. The usual finding is that accuracy decreases as the number of distractor items in the display increases (e.g., Estes \& Taylor, 1966). (This result is limited to the case in which the distractor items are visually confusable with the targets; for instance, very little difference is found when the targets are letters and the distractors are solid rectangles; Estes, 1972.) A category effect in visual detection analogous to that in visual search has been demonstrated by Schneider and Shiffrin (1977), Duncan (1980), and others. When subjects were asked to detect the presence or absence of a digit in a display of letters, relatively little effect of the number of items presented is found.

This result does not seem to depend on the use of letters and digits. For instance, Shiffrin and Schneider (1977) divided a set of letters into a target set and a distractor set. After a great deal of practice with the items, a kind of category effect emerged: Subjects could easily detect a target regardless of the number of distractors present. This suggests that the category effect is not limited to digit and letter categories, but can occur whenever the targets and the distractors come from distinct, well-learned categories.

The third domain in which a category effect has been found is the partial-report task developed by Sperling (1960). In this task, subjects view a brief display, and must report several items identified by a subsequent cue. Performance is typically compared with that in a whole-report condition in which all of the items in the array are to be reported. When the cue specifies the items to be reported in terms of their location or color, partial report has a considerable advantage over whole report. A category effect in this task would consist of a similar partial-report advantage when the cue indicated that only digits or letters were to be reported. It would show that more infor- 
mation was retained when only one category was relevant. Although early research failed to find this category effect (Sperling, 1960; von Wright, 1968), later research did find some advantage for category cues (Dick, 1969; Duncan, 1983; Merikle, 1980; von Wright, 1972).

A number of reasons have been suggested for the failure to find a category effect in the earlier partial-report experiments. These include the form of the visual display (Merikle, 1980), uncertainty about the nature of the cues (Dick, 1971), and the number of cued items (Duncan, 1983). Perhaps the most important variable is whether subjects know the relevant category prior to display onset. For instance, von Wright (1972) apparently found a small category effect when the cue preceded the display, but none when the cue followed the display. Moreover, a similar result is found in visual search. In that task as well, a category effect is found only when subjects have prior knowledge of the relevant category (Gleitman \& Jonides, 1978; Taylor, 1978). It appears that subjects require a certain amount of time to get ready, or prepare, for a particular category, and that without it they cannot take advantage of the category distinction (cf. Brown, 1960).

At least two kinds of explanations have been proposed for the category effect. The first might be called a "partial analysis" explanation (Gleitman \& Jonides, 1976). It proposes that the category of an item can be easily distinguished on the basis of some collection of visual features, and that a partial analysis can isolate the relevant category items before any further processing is required. Thus, when all of the targets are in one category and the distractors in another, subsequent processing can be devoted solely to those items in the target category. The category effect occurs because the distractor items generally do not have to be fully processed.

A number of researchers have found a category effect even after controlling for the more obvious feature differences between targets and distractors (Ingling, 1972; Jonides \& Gleitman, 1972; Shiffrin \& Schneider, 1977). With such control, categorization must be based not on only one or two features, but on a collection of features. The partial-analysis explanation assumes that even if several features must be used to categorize an item, the process would still be faster than a complete analysis of the item. The assumption is that it would be more efficient to categorize an item first, and then to complete the identification only if it is in the relevant category, than it would be to identify all of the items regardless of category.

The second explanation can be called the "parallelcategorization" account. It assumes that all items in a display are identified and categorized in parallel without the use of any processing capacity (Duncan, 1980; Gardner, 1973). Subsequent operations, such as memory rehearsal or item comparisons, would require processing capacity and would normally limit performance. But when target and distractor items belong to different categories, these limitations could be avoided by selectively attending only to items in the relevant category. That is, since items are categorized in parallel prior to any capacity-limited processing, information about category can be used to direct subsequent processing. When all targets belong to the same category, the irrelevant category items can be effectively ignored. Thus, search time and detection accuracy should be unaffected by the number of items in the irrelevant category.

The present work provides some additional constraints on these two classes of explanations. Although the data do not conclusively rule out either one, both of the simple versions outlined above have to be modified to accommodate the results from the present task. Subjects were shown two brief displays separated by a variable stimulusonset asynchrony (SOA). One display was the stimulus array, and the other was the target item. The subjects' task was to decide whether the target item was one of the items in the stimulus array. The stimulus-onset asynchrony between the array and the target could be (1) negative, in which case the target preceded the array, (2) positive, in which case the target followed the array, or (3) zero, in which case the target and array were simultaneous. Similar tasks have been used by Di Lollo and his colleagues (Di Lollo, Lowe, \& Scott, 1974; Di Lollo \& Moscovitch, 1983) and by Townsend (1973).

When the target follows the array, the task is essentially a partial-report task in which subjects report on the basis of item identity. On the other hand, when the target precedes the array, the task becomes a visual detection task in which subjects look for a particular item in the array. A category effect in this task would be demonstrated if performance improved when some of the distractors were from an irrelevant category. In Experiment 1 , a condition in which both the target and the array were digits was compared with one in which all but one of the array items were letters. As indicated above, a critical factor in whether a category effect is found seems to be whether subjects know the relevant category before seeing the display. Accordingly, the subjects were told that the targets in both conditions would be digits.

\section{EXPERIMENT 1}

\section{Method}

Each trial consisted of two brief displays separated by a variable stimulus-onset asynchrony (SOA). One display was the stimulus array, which consisted of a row of seven alphanumeric characters. The other display was the target item. The subject's task was to decide whether the target character matched one of the seven characters in the array, and to press either a "present" or "absent" response button. SOA varied from $-600 \mathrm{msec}$ to $+700 \mathrm{msec}$. By convention, a negative SOA indicates a trial in which the target preceded the stimulus array and a positive SOA indicates a trial in which the target followed the array. At 0 SOA, the two displays were shown simultaneously. Experiment 1 had two conditions. In the digits-only condition, the stimulus array consisted of seven different digits excluding 0 and 1 . In the digit-plus-letters condition, the array consisted of only one digit and six different capital consonants excluding $\mathrm{N}$ and $\mathrm{W}$. In both conditions, the target item was always 
a digit; on "present" trials, it matched an item in the array and on "absent" trials it did not.

The procedure on each trial was as follows. When the computer was ready to start a trial, it displayed a fixation pattern consisting of four dots at the corners of an imaginary rectangle. The subjects initiated each trial by pressing the two response buttons simultaneously. After $500 \mathrm{msec}$, the first display was shown; the second display followed at the appropriate SOA. The fixation pattern remained on the screen until $250 \mathrm{msec}$ after the second display. Between trials, the screen was blank for a variable interval ranging from 200 to $500 \mathrm{msec}$. No feedback was given.

The stimuli were shown on a 30 -cm black-and-white video monitor at a distance of about $70 \mathrm{~cm}$. At that distance, characters subtended $0.4^{\circ}$ of horizontal visual angle. The array was centered on the screen, and the target item was $0.9^{\circ}$ above it. The four dots in the fixation pattern surrounded the target and array positions. Subjects were run in a semi-illuminated room with a space-average luminance of about $9 \mathrm{~cd} / \mathrm{m}^{2}$. The monitor was adjusted so that the characters were displayed at near-maximum contrast against a dark background $[100 \times(\mathrm{L} \max -\mathrm{L} \min ) /(\mathrm{L} \max +\mathrm{L} \min )=95 \%$ contrast]. The space-average luminance of the characters was about $40 \mathrm{~cd} / \mathrm{m}^{2}$. Both the target and the array were shown for a single video raster scan (i.e., they were presented for a nominal duration of $16.7 \mathrm{msec}$ ). The displays were controlled by an Apple II microcomputer system, which also recorded subjects' responses. The characters were presented in the standard font generated by the Apple's video driver.

Both SOA and condition were blocked. Half of the subjects had 10 digits-only blocks followed by 10 digit-plus-letters blocks, and the other half had the reverse order. Each condition began with two blocks of practice, one at 500-msec SOA and the other at -400 -msec SOA, followed by eight blocks with SOAs of $-600,-200,-100$, $0,100,200,300$, and 700 . The order of these eight blocks was balanced across subjects. Each block began with 10 practice trials followed by 50 test trials divided equally between "present" and "absent" trials. A session lasted about $100 \mathrm{~min}$. The subjects were 8 undergraduates at the University of Alberta fulfilling course requirements.

The principal analysis was conducted on a nonparametric measure of sensitivity, $A^{\prime}$, calculated for each subject, condition, and SOA (Grier, 1971; Pollack \& Norman, 1964). A' can be interpreted as the area under the receiver operating characteristic, and is equivaIent to the proportion correct that would result in a two-alternative forced-choice paradigm (Green \& Swets, 1966). However, it might be argued that the assumptions of the theory of signal detection are not valid in this task. Consequently, subsidiary analyses were also conducted using the raw response probabilities.

\section{Results}

$\mathrm{A}^{\prime}$ measures at each SOA and for each condition are shown in Figure 1. The digit-plus-letters condition had significantly better performance than the digits-only condition $[F(1,7)=69.92, p<.001]$. There was also a significant effect of SOA, such that performance was worse with positive SOAs than with negative, and worst of all at $+100 \mathrm{msec}[\mathrm{F}(7,49)=16.46, \mathrm{p}<.001]$. The effect of condition was much larger with positive SOAs (target following the array) than with negative SOAs [target preceding the array, $F(7,49)=4.85, p<.005]$.

Figure 2 shows the percent correct for "present" trials (in which the target was in the array) and "absent" trials (in which the target was not in the array). In general, the results mirrored the $A^{\prime}$ analysis: There was an effect of condition $[F(1,7)=79.57, p<.001]$, an effect of SOA $[F(7,49)=17.76, p<.001]$, and an interaction between

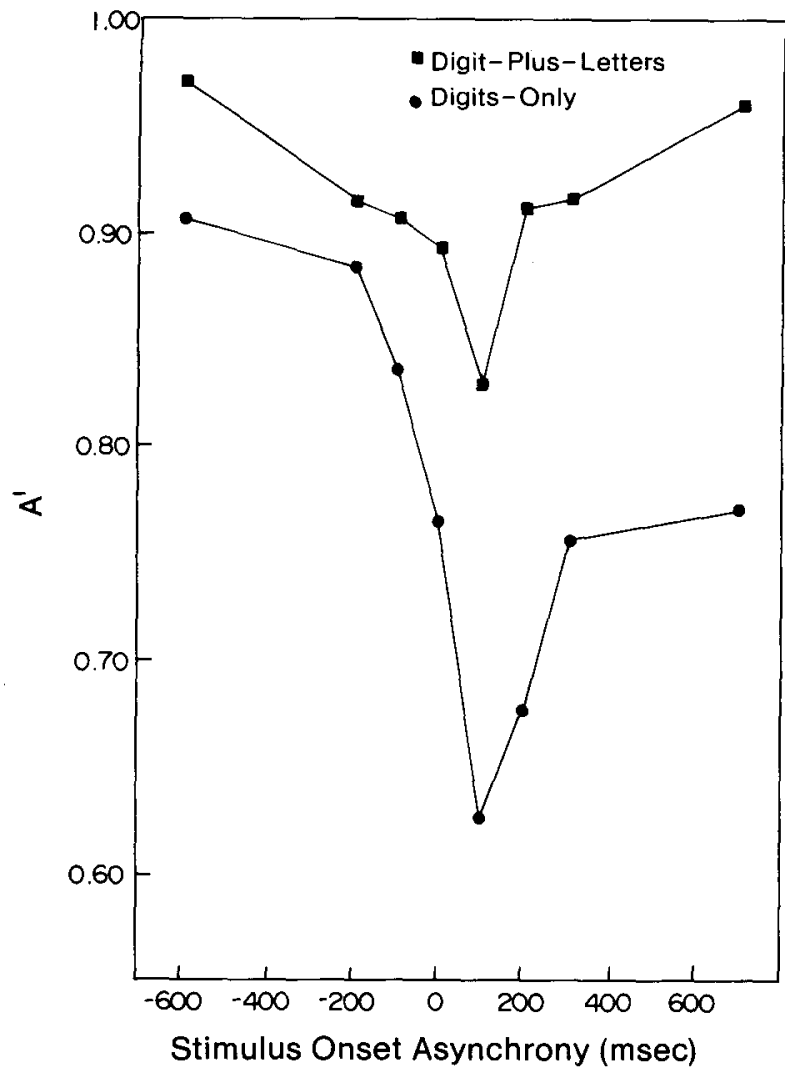

Figure 1. $A^{\prime}$ for the two conditions in Experiment 1 as a function of stimulus-onset asynchrony.

the two $[F(7,49)=5.19, p<.001]$. In addition, the effect of SOA interacted with "present" versus "absent" trials $[F(7,49)=3.84, p<.005]$. As can be seen in Figure 2, the principal cause of the interaction was that the number of correct " absent" responses decreased sharply at SOAs of 100 and $200 \mathrm{msec}$ before recovering at longer SOAs, especially in the digits-only condition. In other words, at short positive SOAs, there were many false alarms in which subjects incorrectly reported the target as being in the array.

Serial position effects were assessed on "present" trials. Table 1 shows a W-shaped serial position curve in which performance was best at the ends and in the middle. The difference across serial positions was significant $[\mathrm{F}(6,42)=3.18, \mathrm{p}<.05]$, as was a contrast comparing the middle and end positions with the other four $[F(1,7)=$ $17.83, \mathrm{p}<.001]$. However, there was no interaction with condition or with SOA.

\section{Discussion}

The two important findings of Experiment 1 were a category effect at both positive and negative SOAs, and a much larger category effect with positive SOAs. These two results are discussed in turn.

The first finding replicates the category effect found in visual detection and partial-report experiments. In the 


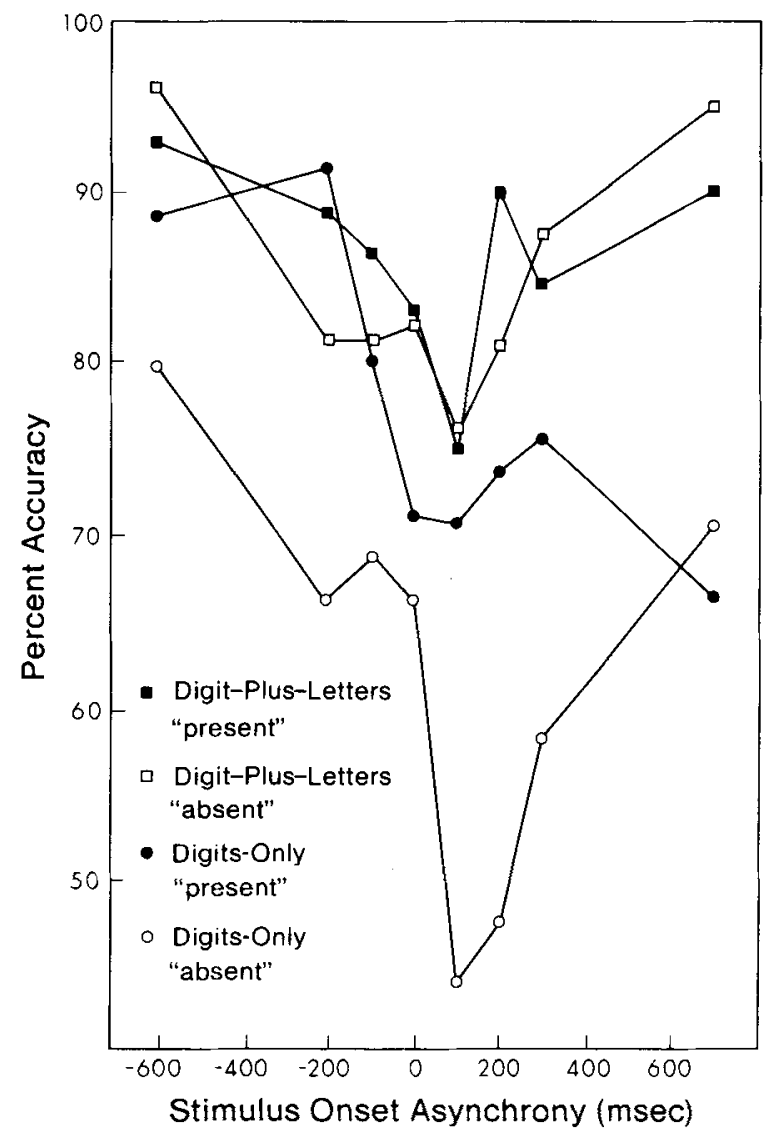

Figure 2. Percent accuracy for "present" and "absent" trials in Experiment 1 as a function of stimulus-onset asynchrony.

negative SOA portion of the curve, where the target item precedes the array, the results demonstrate the same kind of effect found by Duncan (1980), Schneider and Shiffrin (1977), and others. That is, people have less difficulty detecting a particular digit among letters than among other digits. On positive-SOA trials, where the target follows the array, the results show dramatically that a category effect does occur in partial-report tasks. Although this result has been obtained previously (e.g., Duncan, 1983; Merikle, 1980; von Wright, 1972), the present demonstration offers two advantages over at least some of the earlier experiments.

The first is that the present paradigm minimizes output interference. One limit on performance in partial-report tasks is that reporting the first few items interferes with

Table 1

Percent Accuracy for Serial Positions in Experiment 1

\begin{tabular}{cc}
\hline Serial Position & Percent Accuracy \\
\hline 1 & 86.3 \\
2 & 70.4 \\
3 & 83.3 \\
4 & 88.6 \\
5 & 85.5 \\
6 & 74.6 \\
7 & 81.1 \\
\hline
\end{tabular}

one's memory for other items in the display (Dick, 1971). In Experiment 1, this problem is eliminated by requiring subjects to make only a single yes/no response. Output interference in the more traditional partial-report task was also addressed by Duncan (1983). He used a mask to decrease accuracy to one or two items. The reasoning was that if only one or two items were reported, little output interference could occur. However, it is possible that interference was still present; the number of items reported may have been low because of the combined effects of output interference and the degradation caused by the mask. Thus, output interference might have been present even when only one or two items were reported.

A second advantage is that the two conditions are unambiguously comparable. In the traditional partial-report task (e.g., Sperling, 1960), different cues are used on different trials in the partial-report condition, but no cues are used in the whole-report condition. Because of this difference, subjects may use different strategies in the two conditions. Dick (1971) ensured that subjects used the same strategy in both conditions by mixing partial- and whole-report trials within a block of trials. However, von Wright (1972) argues that this biases subjects against using a strategy that would lead to a partial-report advantage. In the present task, the target functions as a type of partialreport cue, since it indicates which item should be reported (cf. Townsend, 1973). However, the nature of this "cue" was precisely the same in the digit-plus-letters and digitsonly conditions. In addition, the qualitative similarity in the results for the two conditions suggests that there were no strategic differences between them. Thus, the two conditions are clearly comparable.

The second major finding of Experiment 1 was that the category effect for partial report was much larger than that for visual detection. The two kinds of effects are directly comparable in the present task, because the same kind of response was required; only the temporal parameters of the display were changed. It might be argued that the smaller category effect found with negative SOAs was due to a ceiling effect: Because sensitivity was fairly high with negative SOAs even in the digits-only condition, there may not have been much room for improvement. However, a close inspection of Figure 1 fails to support such an interpretation. $A^{\prime}$ dropped considerably from -600 -msec SOA to -100 -msec SOA, but the size of the category effect was almost precisely the same at -600 and $-100 \mathrm{msec}$. If a ceiling effect were involved, one would expect the size of the effect to increase as sensitivity dropped further from the ceiling. In addition, the increase in the category effect from 0 to $700 \mathrm{msec}$ was entirely due to an increase in $A^{\prime}$ for the digit-plus-letters condition. Again, this would not be expected if the digitplus-letters condition were subject to a ceiling effect. In sum, the interaction between the category effect and SOA does not seem to be due to any simple scaling artifacts.

Both the partial-analysis hypothesis and the parallelcategorization hypothesis can be elaborated to account for this interaction without too much difficulty. The partial- 
analysis account of the category effect might suggest that the perceptual processing required to detect a particular target is more limited than that required to identify and remember items in an array. In detection, each distractor item in the array must be analyzed only up to the point at which a mismatch with the target is found; for instance, only a single disconfirmatory feature may be required. However, when the array items have to be remembered, each item must be fully processed so that the identity of the item can be found and rehearsed in memory.

This difference in processing would lead to the obtained interaction. In the digit-plus-letters condition, the target item is distinguished by a particular category. Thus, when the target precedes the array, distractors can be dismissed either when they do not match the target or when they are found to be in the irrelevant category, whichever is detected first. Presumably, this takes less time than detecting only target mismatches (as would be the case in the digits-only condition). However, because the processing of each distractor item is already minimal, the advantage may not be great.

A larger advantage would occur when the target follows the array. In the digit-plus-letters condition, only one of the items has to be completely processed, and the rest can be rejected as irrelevant after only a partial analysis. But in the digits-only condition, all of the items must be fully processed and remembered. Assuming the partial analysis is substantially faster than this complete identity analysis, a large category effect would be found. In other words, the category effect may be much larger when the target follows the array because more savings can be had by rejecting irrelevant items.

The parallel-categorization explanation also explains the interaction by appealing to differences in processing with positive and negative SOAs. However, there is less direct interaction with the category effect mechanism. In both halves of the curve, the items are categorized in parallel, and in the digit-plus-letters condition, only a single item is selected for further processing. On the negative SOA trials, the category effect occurs because comparing a single item with the target item is easier than comparing seven items with the target. The difference in accuracy is due to the extra processing needed to compare six additional items in the digits-only condition. On positive SOA trials, the category effect occurs because remembering one item is easier than remembering seven. Thus, the category effect for positive SOAs is due to remembering six additional items, whereas the effect for negative SOAs is due to comparing six additional items. If it is assumed that the memory processes used on positive SOA trials are more difficult than the comparison operations used on negative SOA trials, the obtained interaction is predicted.

A striking feature of the results is the dip in sensitivity at an SOA of 100 . Similar effects were also found by Dixon (in press-a, in press-b) and Di Lollo and Moscovitch (1983). Di Lollo and Moscovitch argued that the dip in accuracy was probably not due to visual mask- ing of the array by the target, since the effect occurred with larger temporal and spatial separations than has usually been found in masking studies. Instead, they proposed an explanation in terms of processing interference: When the target item is presented at a critical point during the processing of the array, the target and the array may compete for processing resources and interference may occur. This account is analogous to interference theories of visual masking (e.g., Turvey, 1973), except that the interference is presumed to occur at a higher level. In any event, the interference seems to be independent of the category effect, in that both conditions show a similar dip in sensitivity.

Another possible account of the dip in sensitivity at short SOAs is that the array masks or interferes with the target item, and that subjects sometimes fail to identify the target correctly. This hypothesis does not conform to subjects' introspections; they generally report that the target is almost always clear and easily perceived. However, to check this possibility, three additional subjects were run in the digits-only condition. These subjects were instructed to write down the target digit after they had made their " "present"'/" absent'" response. The pattern of A' results (shown in Table 2) was similar to the patterns obtained in Experiment 1. Nevertheless, when identifying the target, none of the three subjects made more than a single error. Thus, the errors shown in Table 2 occurred because subjects could not decide whether the target was in the array, not because they failed to identify the target in the first place.

\section{EXPERIMENT 2}

It is puzzling that a category effect of the magnitude found in the present task was not evident in the early work on the partial-report task. As pointed out above, problems with output interference and comparability of the partialand whole-report conditions may have reduced the category effect. However, another possibility has been suggested by several researchers: The failure to find a category effect may be related to the number of items in the relevant category. For instance, Duncan $(1980,1983)$ proposed that items must pass through a limited-capacity system after being identified, and that this system can effectively deal with only one item at a time. Thus, performance in a partial-report condition will decline when there is more than one relevant item. In the same spirit is the

Table 2

$A^{\prime}$ in Target Report Experiment

\begin{tabular}{cc}
\hline $\begin{array}{c}\text { Stimulus-Onset } \\
\text { Asynchrony (msec) }\end{array}$ & $\mathrm{A}^{\prime}$ \\
\hline-600 & .937 \\
-200 & .838 \\
-100 & .886 \\
0 & .817 \\
100 & .756 \\
200 & .711 \\
300 & .781 \\
700 & .811 \\
\hline
\end{tabular}


suggestion made by Shiffrin and Schneider (1977) that attention would be drawn to items in the relevant category, but that with many items the demands on attention would conflict and cancel each other out. Experiment 2 attempted to test the hypothesis that the category effect depends on the number of items in the relevant category.

Francolini and Egeth (1979) investigated this hypothesis in a visual search task. They required subjects to search an array for a particular digit, and measured response time as a function of the number of letter distractors in the array. They compared three conditions in which there were one, two, or three digits in the array in addition to the letter distractors. They found no differences among these conditions, apparently disconfirming the hypothesis that it is the number of items in the target's category that determines whether a category effect occurs. However, this result is difficult to interpret, because Francolini and Egeth did not find a category effect in any of the conditions. In visual search, the category effect is the finding that the number of distractors has a much smaller effect when the target and distractors come from different categories. Francolini and Egeth found the same small effect of the number of letter distractors with digit targets and letter targets, and failed to find a category effect even when there was only a single array item from the relevant category. Thus, the hypothesis was not tested under conditions that produce a clear category effect.

Duncan (1983) tested the hypothesis by varying the number of relevant items in a partial-report task. In one condition, the array consisted of one digit and five letters; in another condition, it consisted of three digits and three letters. Performance in a partial-report condition (in which subjects reported only digits) was compared with that in a whole-report condition (in which all items were reported). Consistent with the hypothesis that the number of relevant items is critical, Duncan found a substantial partial-report advantage with one relevant item, but a much smaller advantage with three relevant items.

Experiment 2 attempted to replicate this result using the target-array task from Experiment 1 . The effects of number of relevant items could then be considered in both partial report and visual detection. Experiment 2 used the same task and stimuli as Experiment 1, but there were three items in the relevant category instead of one. If the category effect is unrelated to the number of relevant items, the category effect should be obtained here just as in Experiment 1. On the other hand, if it depends critically on there being only a single relevant item, no category effect should be obtained.

A very similar experiment was performed by Di Lollo and Moscovitch (1983). Their stimulus array contained six items, and in one condition half of the items were letters and half were digits. Subjects knew in advance whether the target was to be a letter or a digit. They found a consistent advantage for the letter/digit arrays relative to arrays of all letters or all digits. However, their experiment may not have been a fair test of the category effect, because the items in a particular category were always grouped together (e.g., "XMT435'). Thus, after identifying one item, the subjects would have been able to decide whether the left or right side of the display contained the target and to attend to that side alone. In a sense, the advantage for mixed letter and digit arrays found by Di Lollo and Moscovitch may have been due to selective attention to location rather than category. In the present task, the items in the two categories were randomly intermixed to avoid this possibility.

\section{Method}

The apparatus and procedure were identical to those in Experiment 1 . There were two stimulus conditions: letters only and letters plus digits. The letters-only condition was similar to the digitsonly condition of Experiment 1; the stimulus array contained seven different letters from the set of consonants excluding $\mathrm{N}$ and $\mathrm{W}$. The letters-plus-digits condition had stimulus arrays containing three letters and four digits in a random order. The digits were chosen from the digits 2-9, as in Experiment 1. The target item was a letter in both conditions.

Because of difficulty in scheduling subjects for a single long session, the subjects were run in two 50 -min sessions on successive days. Each session contained eight blocks of trials, one each using the SOAs $-600,-200,-100,0,100,200,300$, and $700 \mathrm{msec}$. Blocks of letters-only trials alternated with blocks of letters-plusdigits trials. The order in which the SOAs were run was random in the first session. The second session used the same order of SOAs, but began with the opposite condition, so that each SOA was run in both conditions. Each block consisted of 10 practice trials followed by 50 test trials divided equally between "present" and "absent" trials. The subjects were 8 undergraduates at the University of Alberta fulfilling course requirements.

\section{Results}

The $A^{\prime}$ results are shown in Figure 3. As in Experiment 1 , there was a significant effect of SOA caused by poorer performance at positive SOAs, especially at 100 and $200 \mathrm{msec}[\mathrm{F}(7,49)=28.32, \mathrm{p}<.001]$. However, there was no difference between conditions $[\mathrm{F}(1,7)<1]$ and no interaction between condition and SOA $[F(7,49)$ $<1$ ]. A $95 \%$ confidence interval for the category effect in this experiment was estimated to be -0.026 to +0.016 . In comparison, the overall size of the category effect in Experiment 1 was 0.134 . Thus, if a category effect occurred in the present experiment, it is unlikely to have been much more than 1/10th the size of that in Experiment 1.

Figure 4 shows the accuracies for "present" and " $a b$ sent" trials. There was a significant effect of SOA $[F(7,49)=40.36, p<.001]$ and an interaction between type of trial and $\operatorname{SOA}[F(7,49)=2.22, p<.05]$. As in Experiment 1 , the dip in overall accuracy at $100-200 \mathrm{msec}$ was almost entirely due to performance on "absent" trials. However, as with the $\mathrm{A}^{\prime}$ analysis, there was no effect or interaction involving condition.

\section{Discussion}

The results showed no evidence of a category effect when there were three items in the array from the relevant category. In conjunction with the results from Experiment 1 , these results suggest that a category effect 
occurs only when there is a single relevant item. The results support the hypotheses advanced by Duncan (1980, 1983) and Shiffrin and Schneider (1977) that some processing component in the task can effectively deal with only a single item at a time.

A number of objections might be made to this conclusion. One is that the relevant category here was letters, whereas the relevant category in Experiment 1 was digits. Thus, the two experiments may not be directly comparable. Second, a linear array of items was used, whereas a circular array was used in several experiments that found a partial-report advantage (e.g., Duncan, 1983; Merikle, 1980). Thus, the null result found here may be related in some way to the use of a linear array. In order to counter these objections, Experiment 2 was replicated with a circular array and with digits as the relevant category. The array consisted of three digits and four letters arranged at equal intervals around the perimeter of a $1.1^{\circ}$-diam circle. The target was always a digit and appeared in the center of the circle. Mean A' measures for 11 subjects are shown in Table 3. The pattern of results was quite similar to that of Experiment 2. In particular, neither the effect of condition nor the interaction of condition and SOA was significant. Thus, the results of Experiment 2 do not seem to depend on the use of letters as a relevant category or on the use of a linear array.

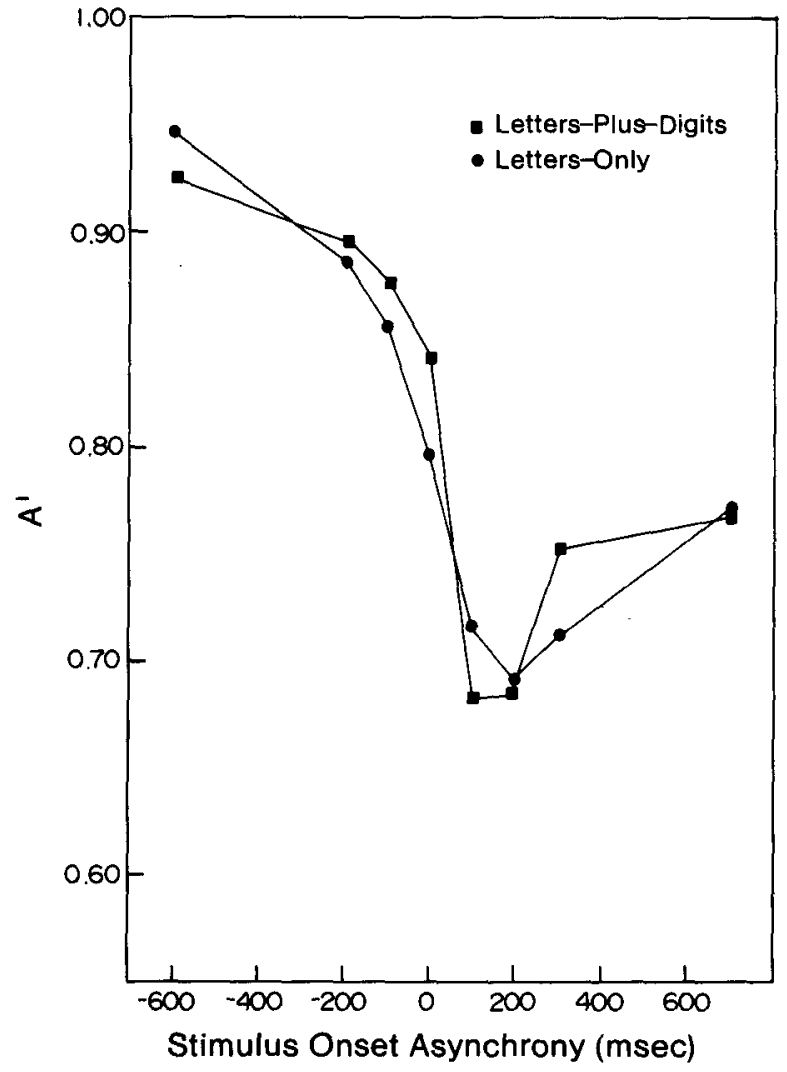

Figure 3. $A^{\prime}$ for the two conditions in Experiment 2 as a function of stimulus-onset asynchrony.

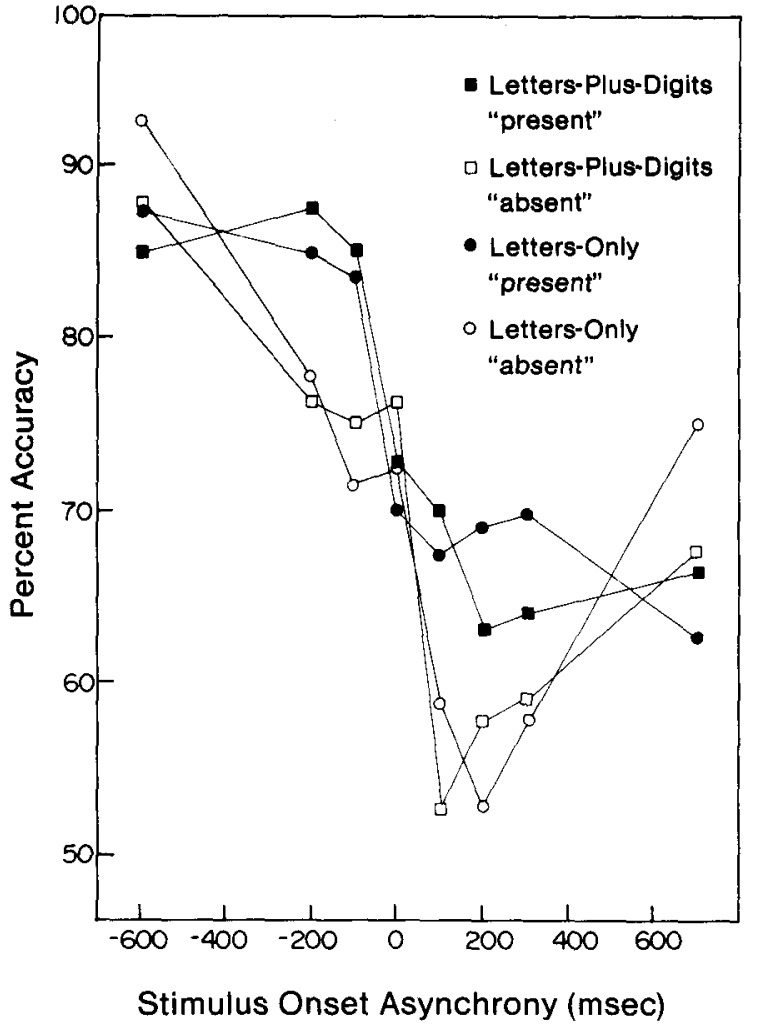

Figure 4. Percent accuracy for "present" and "absent" trials in Experiment 2 as a function of stimulus-onset asynchrony.

Another possible objection is that the blocking of trials was not carried out in the same way in Experiments 1 and 2 . In the first experiment, all of the blocks for a given condition were grouped together, whereas in the second experiment, the two conditions alternated from block to block. Thus, subjects in the first experiment may have had more opportunity to practice the preparations or strategies necessary to produce the category effect. To counter this objection, Experiment 1 was replicated with trials from both the digits-only and digit-plus-letters conditions mixed together in a block. Because subjects could not anticipate the nature of each trial, they would be unlikely to adopt a strategy appropriate for one particular condition. As in the previous replication, the array was presented around the perimeter of a circle with the target appearing at the center.

The $\mathrm{A}^{\prime}$ results from 16 subjects are shown in Table 4. Although the size of the category effect is somewhat smaller than in Experiment 1, the pattern of results is quite similar. There was an overall difference between the digits-only and the digit-plus-letters conditions $[\mathrm{F}(1,15)=$ $27.64, \mathrm{p}<.001$ ], and the effect was larger with positive SOAs than with negative SOAs $[F(7,105)=4.95$, $p<$ $.001]$. The overall effect of SOA was also significant $[F(7,105)=38.15, p<.001]$. These results are consistent with those of a visual search experiment conducted by Gleitman and Jonides (1978). They found a category 
Table 3

$A^{\prime}$ in Experiment 2 Replication

\begin{tabular}{ccc}
\hline $\begin{array}{c}\text { Stimulus-Onset } \\
\text { Asynchrony (msec) }\end{array}$ & Digits Only & Digits Plus Letters \\
\hline-600 & .948 & .945 \\
-200 & .935 & .944 \\
-100 & .910 & .885 \\
0 & .832 & .826 \\
100 & .743 & .746 \\
200 & .637 & .787 \\
300 & .768 & .768 \\
700 & .764 & .784 \\
\hline
\end{tabular}

Table 4

$A^{\prime}$ in Experiment 1 Replication

\begin{tabular}{ccc}
$\begin{array}{c}\text { Stimulus-Onset } \\
\text { Asynchrony (msec) }\end{array}$ & Digits Only & Digit Plus Letters \\
\hline-600 & .944 & .953 \\
-200 & .913 & .936 \\
-100 & .869 & .928 \\
0 & .856 & .878 \\
100 & .760 & .789 \\
200 & .700 & .779 \\
300 & .691 & .823 \\
700 & .754 & .854 \\
\hline
\end{tabular}

effect on an unexpected mixed-category trial that subjects could not have prepared for at all. Thus, it seems unlikely that any special strategy for mixed-category trials is needed to produce the effect.

Neither the partial-analysis explanation nor the parallelcategorization explanation can account for the results of Experiment 2 in the simple form described above. In the partial-analysis explanation it was assumed that an efficient mechanism could consider features of each item and dismiss most of them because they were not in the relevant category. Such a mechanism should be able to operate regardless of the number of relevant items. When there are three items instead of one, fewer can be dismissed as part of the irrelevant category, and the category effect should be smaller. But even if the effect is a great deal smaller than that found in Experiment 1, it should still have been observable. A similar argument can be made for the parallel-categorization explanation. If the categorization were perfect and the selection of the relevant items automatic, performance with three relevant items should be the same as with an array of only three items. This would be considerably easier than an array of seven items at all SOAs (Di Lollo \& Moscovitch, 1983). Even if categorization were not perfect, performance should still have been better with three relevant and four irrelevant items than with seven relevant items.

Both accounts can be modified to fit these results by assuming that selecting the relevant items for further processing requires some effort. Thus, using category information to select only the relevant items would have a cost as well as a benefit. When there is only one item in the relevant category, the cost of attending to that item alone would be small relative to the advantage of not having to process all of the items. On the other hand, when three of seven items are in the relevant category, the cost in selecting and attending to all three would be correspond- ingly greater, but the number of items that could be ignored would be much smaller. Thus, the strategy of attending only to items in the relevant category would be much more efficient in Experiment 1 than in Experiment 2 . In the second experiment, the cost of selecting and attending to the three relevant items may have been so great that it was easier simply to try to operate on as much of the array as possible, without considering item category. In other words, the cost of selecting the items in one category would have outweighed the advantages, leading subjects to abandon that strategy.

The results of Experiment 2, then, suggest that both the parallel-categorization and the partial-analysis explanations of the category effect require something akin to a selective-attention mechanism to decide which items should be processed further. In both cases, the decision is based on item-category information that is acquired with a minimum of processing effort. Furthermore, although this mechanism can operate effectively when there is only one item to be selected, it functions much less effectively when there are three relevants items to be selected. In fact, the results suggest that there is no category effect at all with three relevant items. The only difference between the two accounts at this point is the way in which information about item category is obtained. In the partialanalysis account, it is obtained by minimal analysis of visual features; in the parallel-categorization account, it is obtained after items have been automatically categorized.

This account suggests an alternative interpretation of the results of Di Lollo and Moscovitch (1983). In their Experiment 3, arrays consisting of three letters and three digits led to much higher accuracies than did arrays of six letters or six digits. The most important difference between their study and the present one was that items in each category were grouped together (e.g., "XMT435"). This may have allowed subjects to select out a group of items for further processing, as found by Treisman (1982) in visual search. Thus, even though subjects find it difficult to attend separately to three different items, they may be able to attend to a single group containing three items.

Besides the grouping, the only other substantive difference between the Di Lollo and Moscovitch (1983) displays and those used here was that their characters were about twice as large, and were displayed for $2 \mathrm{msec}$ instead of $16.7 \mathrm{msec}$. In addition, Di Lollo and Moscovitch's subjects were somewhat more practiced and received more feedback. However, the category effect found in this task is extremely robust; for example, all of the subjects in Experiment 1 showed the effect. Thus, it is unlikely that these minor differences were responsible for the failure to find a category effect in Experiment 2.

\section{GENERAL DISCUSSION}

The experiments reported here replicated the category effect in visual detection and partial report, and demonstrated two additional results: When other aspects of the 
tasks are equated, the category effect is larger with partial report than with visual detection, and when there are three relevant items instead of one, the category effect seems to disappear. Although both the partial-analysis explanation and the parallel-categorization explanation can provide plausible accounts of these results, they both may require the addition of an effortful selective-attention mechanism to explain why no category effect was found with three relevant items.

Two other explanations of the category effect should also be discussed. The first is the "final transfer" theory described by Duncan $(1980,1983)$. He divided the processing of letters and digits into a preattentive analysis and a limited-capacity system. Items were assumed to be fully identified at the preattentive level, but were required to pass through the limited-capacity system one item at a time before they became available for comparison or report. In this model, the category effect arises because information about item category can be used to select items for the limited-capacity system, and so items from the irrelevant category do not compete for the limited-capacity system. However, the effect may be small, because category information is derived slowly or inaccurately and is not completely effective at guiding the selection.

The principal difference between the final-transfer theory and the parallel-categorization model is in how the number of items in the relevant category affects performance. Duncan (1983) suggested that the category effect should decrease as the number of relevant items increases because more items are competing for access to the limited-capacity system. However, there is no reason to suspect that the category effect should disappear entirely with several relevant items. The parallel-categorization account developed here hypothesizes that, with three relevant items, subjects may make a strategic choice not to attend to the relevant category. They presumably find a strategy of attending to all of the items in the display more efficient. (This leaves open the possibility that under some circumstances subjects may find it more efficient to attend to the three relevant items; the choice of strategy may depend on the discriminability of the items, the number of distractors, and the level of practice.) In the present results, a choice between the two models rests on the question of whether the data in Figure 3 actually represent no category effect, or whether there was a small difference that could not be detected statistically. A definitive answer to this question must await further research.

Another explanation of the category effect was suggested by Deutsch (1977) and elaborated by Taylor (1978) and Kahneman and Treisman (1983). The proposal is that when subjects expect a target in a particular category, detectors in the relevant category are primed or facilitated, and those in the irrelevant category are inhibited. Thus, when a target is presented in the context of irrelevantcategory distractors, the target item will be identified much more quickly than the distractors. There would be no advantage when the distractors were also in the relevant category, since they would be subject to the same priming as the target.

One interpretation of this account is that priming a particular category simply changes the bias to make a response to items in that category. On this interpretation, priming should lead to an increase in false alarms to distractors in the same category. This result was found by Gleitman and Jonides (1976) in a visual search task, but Duncan (1980) showed that a change in bias could not account for the category effect in visual detection. Nor is there evidence in Experiment 1 of the present report that the category effect is accompanied by an increase in false alarms. Thus, a simple form of the priming explanation does not seem to account for these results.

Neither the partial-analysis nor the parallel-categorization accounts predict the large number of false alarms that were found at short positive SOAs (see Figures 2 and 4). Di Lollo and Moscovitch (1983) proposed an interference explanation that can be applied to this result: Presenting the target shortly after the array may interfere with the array processing, leaving the observer with only incomplete or distorted information about the items. One might speculate that observers have a bias to respond "present" when faced with such a large amount of incomplete array information. This would account for the large number of false alarms at SOAs of 100-200 msec. However, in the digit-plus-letters condition of Experiment 1 , the number of false alarms at short SOAs was much less. Thus, the bias for "present" responses may not operate when category information limits the number of relevant items to one.

This kind of interference explanation may be more compatible with partial analysis than with parallel categorization. In the partial-analysis account, category information might be extracted prior to any interfering effects of the probe. Thus, the item in the relevant category could be isolated even if the probe presentation disrupted further processing of the array items. On the other hand, it is more difficult to see how the interference explanation fits with the parallel-categorization account. For instance, in some parallel-categorization theories, item category is determined only after the item is identified. Thus, anything that interferes with the processing of items should also interfere with the processing of category information (cf. Taylor, 1978). And without the category information, there should still be a large number of false alarms in the digit-plus-letters condition.

In sum, both the partial-analysis and parallel-categorization explanations can be expanded to handle the main results of Experiments 1 and 2. However, the large number of false alarms observed in this task at short positive SOAs poses an additional constraint. The interference explanation for this result suggested by Di Lollo and Moscovitch (1983) seems more compatible with the partialanalysis account. 


\section{REFERENCES}

Atkinson, R. C., Holmgren, J. E., \& Juola, C. F. (1969). Processing time as influenced by the number of elements in a visual display. Perception \& Psychophysics, 6, 321-326.

BraND, J. (1971). Classification without identification in visual search. Quarterly Journal of Experimental Psychology, 23, 178-186.

BROWN, J. (1960). Evidence for a selective process during perception of tachistoscopically presented stimuli. Journal of Experimental Psychology, 59, 176-181.

Deutsch, J. A. (1977). On the category effect in visual search. Perception \& Psychophysics, 21, 590-592.

Dick, A. O. (1969). Relations between the sensory register and shortterm storage in tachistoscopic recognition. Journal of Experimental Psychology, 82, 279-284.

Dick, A. O. (1971). On the problem of selection in short-term visual (iconic) memory. Canadian Journal of Psychology, 25, 250-263.

Di Lollo, V., Lowe, D. G., \& SCOTT, J. P., JR. (1974). Backward masking and interference with the processing of brief visual displays. Journal of Experimental Psychology, 103, 934-940.

Di Lollo, V., \& Moscovitch, M. (1983). Perceptual interference between spatially separate sequential displays. Canadian Journal of Psychology, 37, 414-428.

DixoN, P. (in press-a). Attention and interference in the perception of brief visual displays. Journal of Experimental Psychology: Human Perception and Performance.

Dixon, $P$. (in press-b). Stimulus duration may affect partial report performance. Perception.

DunCan, J. (1980). The locus of interference in the perception of simultaneous stimuli. Psychological Review, 87, 272-300.

DunCan, J. (1983). Perceptual selection based on alphanumeric class: Evidence from partial reports. Perception \& Psychophysics, 33, 533-547.

Egeth, H., Atkinson, J., Gilmore, G., \& Marcus, N. (1973). Factors affecting processing mode in visual search. Perception \& Psychophysics, 13, 394-402.

EGETH, H., JonIDES, J., \& WALL, S. (1972). Parallel processing of multielement displays. Cognitive Psychology, 3, 674-698.

EsTEs, W. K. (1972). Interactions of signal and background variables in visual processing. Perception \& Psychophysics, 13, 278-286.

ESTES, W. K., TAYLOR, H. A. (1966). Visual detection in relation to display size and redundancy of critical elements. Perception \& Psychophysics, 1, 9-16.

Francolini, C. M., \& Egeth, H. E. (1979). Perceptual selectivity is task dependent: The pop-out effect poops out. Perception \& Psychophysics, 25, 99-110.

GARDNER, G. T. (1973). Evidence for independent parallel channels in tachistoscopic perception. Cognitive Psychology, 4, 130-155.

Gleitman, H., \& Jonides, J. (1976). Cost of categorization in visual search: Incomplete processing of target and field items. Perception \& Psychophysics, 20, 289-298.
Gleitman, H., \& Jonides, J. (1978). The effect of set on categorization in visual search. Perception \& Psychophysics, 24, 361-368.

GreEN, D. M., \& SWETS, J. A. (1966). Signal detection theory and psychophysics. New York: Wiley.

GrIER, J. B. (1971). Nonparametric indexes for sensitivity and bias: Computing formulas. Psychological Bulletin, 75, 424-429.

INGLING, N. W. (1972). Categorization: A mechanism for rapid information processing. Journal of Experimental Psychology, 94, 239-243.

JoNidEs, J., \& GLEITMAN, H. (1972). A conceptual category effect in visual search: $O$ as letter or as digit. Perception \& Psychophysics, $12,457-460$.

Kahneman, D., \& Treisman, A. (1983). Changing views of attention and automaticity. In R. Paramusen, R. Davies, \& J. Beatty (Eds.), Varieties of attention. New York: Academic Press.

KRUEGER, L. E. (1984). The category effect in visual search depends on physical rather than conceptual differences. Perception \& Psychophysics, 35, 558-564.

MeruKLE, P. M. (1980). Selections from visual persistence by perceptual groups and category membership. Journal of Experimental Psychology: General, 109, 279-295.

Nickerson, R. S. (1966). Response times with a memory-dependent decision task. Journal of Experimental Psychology, 72, 761-769.

Pollack, I., \& Norman, D. A. (1964). A nonparametric analysis of recognition experiments. Psychonomic Science, 1, 125-126.

SCHNEIDER, W., \& SHIFFrIN, R. M. (1977). Controlled and automatic human information processing: I. Detection, search and attention. Psychological Review, 84, 1-66.

SHIFfrin, R. M., \& SCHNEIDER, W. (1977). Controlled and automatic human information processing: II. Perceptual learning, automatic attending, and a general theory. Psychological Review, 84, 127-190.

SPERLING, G. (1960). The information available in brief visual presentation. Psychological Monographs, 74(11, Whole No. 498).

TAYLOR, D. A. (1978). Identification and categorization of letters and digits. Journal of Experimental Psychology: Human Perception \& Performance, 4, 423-439.

TownSEND, V. M. (1973). Loss of spatial and identity information following a tachistoscopic exposure. Journal of Experimental Psychology, 98, 113-118.

Treisman, A. (1982). Perceptual grouping and attention in visual search for features and for objects. Joumal of Experimental Psychology: Human Perception \& Performance, 8, 194-214.

TURVEY, M. T. (1973). On peripheral and central processes in vision: Inferences from an information-processing analysis of masking with patterned stimuli. Psychological Review, 80, 1-52.

VON WRIGHT, J. M. (1968). Selection in visual immediate memory. Quarterly Journal of Experimental Psychology, 20, 62-68.

VON Wright, J. M. (1972). On the problem of selection in iconic memory. Scandinavian Journal of Psychology, 13, 159-171.

(Manuscript received April 23, 1985; revision accepted for publication August 26, 1985.) 